\title{
Essai de caractérisation de la veine verte du merisier
}

\author{
H. POLGE \\ I.N.R.A., Station de Recherches sur la Qualité des Bois \\ Centre de Recherches forestieres, Champenoux, F 54280 Seichamps
}

\begin{abstract}
Résumé
L'étude porte sur 16 échantillons de veine verte (V), 16 échantillons prélevés à proximité $(\mathrm{P})$ et 16 témoins provenant de planchettes sans veine verte $(\mathrm{T})$. Elle montre que les échantillons $\mathrm{V}$ ont un fort pourcentage de bois de tension, et se différencient également par des valeurs élevées pour 4 autres caractéristiques : retrait longitudinal, rendement en fibres, longueur de fibres et vitesse des ultrasons. Les échantillons $\mathbf{P}$ ont des valeurs intermédiaires, mais se rapprochent plus des échantillons $\mathrm{V}$ pour le rendenent, pour la longueur des fibres et pour la vitesse des ultrasons, et des témoins pour le retrait. La densité du bois ne discrimine pas les 3 lots, et est indépendante des autres critères qui sont au contraire très liés entre eux. Une corrélation très significative existe notamment entre la vitesse des ultrasons et la longueur des fibres. Si veine verte et bois de tension sont associés au niveau statistique moyen, il existe au niveau individuel des exceptions dans les 2 sens.
\end{abstract}

\section{Introduction}

La veine verie est un défaut grave du bois de merisier, qui le rend impropre aux usages nobles, placage ou ébénisterie en bois massif, pour lesquels la coulcur naturelle rose est, au même titre que la finesse du grain, un critère essentiel de choix. L_a coloration verdâtre qui apparaît par bandes ne peut être corrigée, et se trouve même accentuée, dans les cas, très fréquents pour le bois de merisier, de teinture artificielle.

Cependant, malgré les incidences financières importantes qu'entrainnent déclassements et réfactions, la littérature forestière est à peu près muette sur le sujet : MASSET (1977) signale la grande importance du problème (qui affecte 20 à 25 p. 100 des merisiers français) et rend compte de l'influence possible, mais non démontrée, de certains facteurs : gel, abondance des précipitations, sols argileux ou marneux mal drainés, voire même hérédité (sensibilité particulière des merisiers de provenance yougoslave). Le premier, semble-t-il, Ferrand (1982) cherche à mettre en relation la vcine verte avec la structure anatomique du bois, en montrant, mais sur un seul échantillon, qu'elle est en fait formée de cellules à couche gélatineuse caractéristiques du bois de tension. Le but du présent travail est de vérifier ce résultat sur un plus large échantillonnage, et également de voir si d’autres propriétés du bois de tension sappliquent aussi à la veine verte. 


\section{Matériel et méthodes}

Le matériel étudié nous a été aimablement fourni par M. HublerT, Secrétaire du groupe de travail Merisier à Institut pour le Développement Forestier; il consistait en 5 petites planches à veine verte ei autant de témoins, dont on ne sait malheureusement pas de combien darbres différents elles pouvaient provenir; dans les premières, ont été découpées, en nombre variable par planchette en fonction de la densité et de la netteté des zones colorées, un total de 16 barrettes dont la longueur (de 9 à $22 \mathrm{~cm}$ ) et la section (de 42 à $88 \mathrm{~mm}^{\circ}$ ) étaient déterminées de façon qu'elles ne soient formées presqu'exclusivement que de veine verte, et 16 autres barrettes, situées à proximité immédiate des précédentes, mais à peu près exemptes de ce défaut. Enfin un troisième ensemble de 16 barrettes a été cébitć cans les planchettes témoins. L'étude porte done sur 48 échantillons se répartissant à égalité entre 3 modalités : veine verte (référence $\langle V »)$, à proximité de veine verte $(\langle P »)$, et témoin $(\langle T\rangle)$; la variabilité du nombre de barrettes par planchette ne permet pas une analyse à ce nivealu, qui d'ailleurs ne présenterait pas dintérêt particulier, sagissant d"établir des liaisons générales entre le céfaut étudié et certaines propriétés anatomiques, physiques ou mécaniques du bois. l.es caractères mesurés sont les suivants :

- Pourcentage die bois de tension :

Il a été déterminé par champs successifs sur coupes microscopiques transversales représentant la totalité de la section des barrettes, après double coloration safranine-bleu Astra ; les mesures ont été faites à l'ana'yseur d'images (Ferrand, 1981) au grossisscment 2.5, avec interposition d'un filtre rouge (la couche gélatineuse, riche en cellulose. apparaît alors en noir sur l’image seuillée).

\section{- Retrait longitudinal :}

Il a été calculé à partir de la mesure de la longueur des barrettes faite successivement à l'état saturé, puis à l'état dìt «sec à laair», stabilisé aux environs de 10 p. 100 d'humidité.

\section{- Densité à l'état sec à l'air :}

Obtenue par rapport du poids des barrettes à leur volume déterminé géométriquement après stabilisation.

\section{- Rendement en fibres :}

Il a été estimé égal au rapport du poids anhydre de fibres libérées par procéclé Kraft au poids anhydre de bois initial ; la technique utilisće est celle des microcuissons (JANIN, 1972), avec un taux dalcali actif de 22, une sulfidité de 25 et un rapport liqueur/bois de 4; le matériel consistait en petits parallélépipèdes découpés à une extrémité des barrettes et représentant environ un gramme de matière.

\section{- Longueur moyenne des fibres :}

Elle a été déterminée à l'aide de l'appareil breveté « Histofibre: (JANIN, 1981), en utilisant une suspension très diluée des fibres obtenues lors de l'essai précédent ; 10000 
fibres ont été mesurées par échantillon, soit un total de 480000 pour l'ensemble de l'expérience (dans un temps inférieur à 2 jours, y compris les opérations annexes, notamment le nettoyage soigné de lappareil entre échantillons).

- Vitesse longitudinale des ultrasons :

Elle a été obtenue à partir du temps mis par des ondes longitudinales émises à la fréquence de $80 \mathrm{KHz}$ pour traverser dans le sens du fil du bois les barrettes utilisées pour les autres essais (BUCUR, 1981) ; cette caractéristique est directement liéc au module d’élasticité du bois.

\section{Résultats}

\subsection{Analyses de variance}

Les calculs ont été faits en prenant, naturellement, comme source de variation, la catégorie de bois ( $V, P$ ou $T$ suivant les références indiquées plus haut). Leurs résultats sont donnés dans le tableau 1 :

\section{TABLend 1}

Analyses de variance.

Variance analysis.

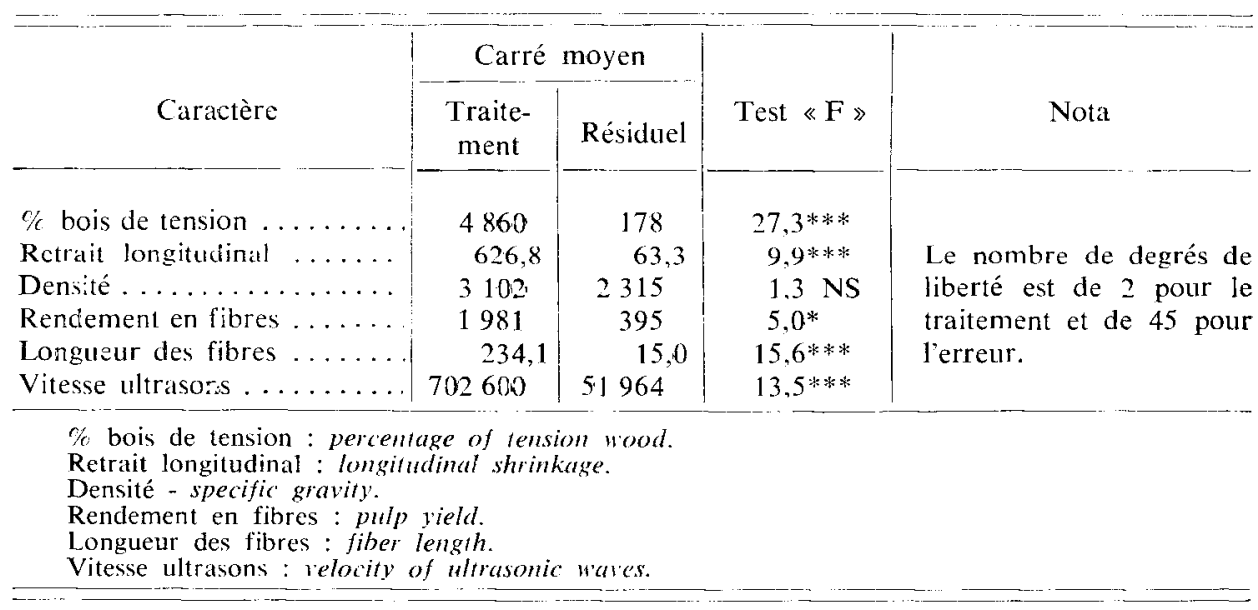

Il existe des différences significatives au seuil de 1 pour 1000 pour toutes les variables, sauf le rendement en fibres (différence au seuil de 5 p. 100), et, surtout, la densité (différence non significative) ; ce dernier résultat doit être noté car il est exceptionnel, la densité du bois apparaissant le plus souvent comme un critère très discriminant et, de plus, lié aux autres caractéristiques par des corrélations très étroites.

Les tests de comparaison de moyennes donnent les résultats suivants : 


\section{TABleau 2}

Tests de comparaison des moyennes.

Tests of comparison of the means.

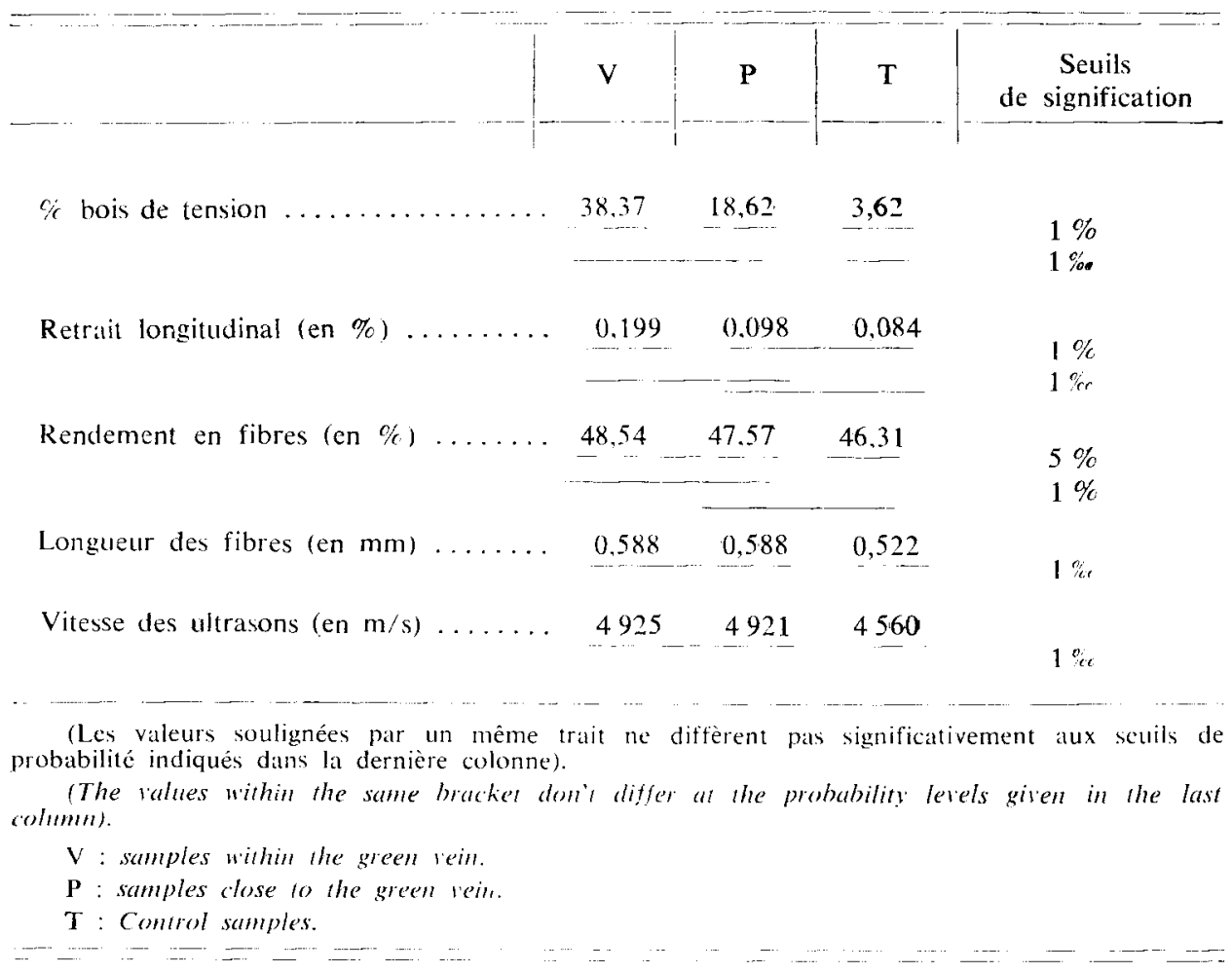

Il se confirme tout d'abord que la veine verte renferme une tencur très élevée de bois de tension (un pourcentage de 69 p. 100 est même atteint dans un échantillon, ce qui signifie, si lon tient compte du fait que vaisscaux et cellules de parenchyme représentent environ 30 p. 100 du volume total, que pratiquement toutes les fibres ont des membranes à couche gélatineuse). Une caractéristique bien connue du bois de tension est son retrait longitudinal élevé ; tel est également le cas pour les échantillons « $V$ ». Enfin, leur fort rendement en fibres confirme les liens entre veine verte ct bois de tension, puisque la couche supplémentaire qui caractérise les parois cellulaires de celui-ci est de nature cellulosique.

Les résultats déjà publiés par ailleurs au sujet des relations entre bois de tension et longueur des fibres sont contradictoires (Hughes, 1965); celle supérieure trouvée dans la veine verte n'infirme pas l'hypothèse d'une relation étroite entre elle et le bois de tension car la majorité des auteurs font état de fibres plus longues dans celui-ci que dans le bois normal. 
Aucune donnée n'existe clans la littérature au sujet de l'influence du bois de tension sur la vitesse des ultrasons; le résultat mentionné ici, faisant apparaître une vitesse dans la veine verte nettement supérieure à celle des témoins, est cependant à rapprocher de celui obtenu sur le hêtre par Gonet (1971) faisant apparaître un module d'élasticité statique du bois de tension supérieur de plus de 11 p. 100 à celui du bois normal, puisqu'il existe une très bonne liaison entre module statique et module dynamique, et puisque celui-ci, à densité du bois égalc, varie comme le carré de la vitesse des ultrasons; une tentative dexplication de la relation vitesse-veine verte sera avancée plus loin.

Globalement, il faut retenir de la comparaison des moyennes :

- que, sauf un cas d'égalité absolue, le bois des échantillons proches de la veine verte possède des caractéristiques intermédiaires entre celui prélevé dans la veine elle-même et les témoins,

— que ces échantillons « $P$ » se rapprochent davantage de la catégoric « $T$ » par le retrait longitudinal, et de la catégorie 《 $V 》$ au contraire par la longueur des fibres et la vitesse des ultrasons.

\subsection{Analyse en composantes principales}

Elle a été entreprise au vu de la bonne séparation des traitements dans les analyses de variance, et des groupements différents qui apparaissent suivant les variables étudiées.

Les pourcentages de l'inertic totale absorbés par les composantes successives sont les suivants :

\section{Tableau 3}

Analyse en composantes principales.

Pourcentages de linertie totale absorbés par les composantes.

Principal component analysis.

Percentages of the total inertia due to the components.

\begin{tabular}{c|c|c|c|c|c}
\hline \multicolumn{1}{c|}{$\mathrm{N}^{\circ}$ des composantes } & 1 & 2 & 3 & 4 & 5 \\
\hline Inertie $\ldots \ldots \ldots \ldots \ldots \ldots \ldots$ & 2,947 & 1,269 & 0,779 & 0,541 & 0,270 \\
Pourcentage $\ldots \ldots \ldots \ldots \ldots \ldots$ & 49,11 & 21,16 & 12,98 & 9,01 & 4,50 \\
$\%$ cumulé $\ldots \ldots \ldots \ldots \ldots \ldots$ & 49,11 & 70,27 & 83,25 & 92,26 & 96,76 \\
\hline
\end{tabular}

La valeur explicative des composantes ne diminue que de façon très progressive, et les 4 premières tout au moins concourent de façon notable à différencier les traitements.

Les coefficients de corrélation entre composantes principales et variables initiales sont donnés dans le tableau 4 : 
TABLEaU 4

Coefficients de corrélation entre composantes principales et variables initiales. Correlation coefficients between the principal components and the initial variables.

\begin{tabular}{|c|c|c|c|c|c|}
\hline $\mathrm{N}^{*}$ des composantes & 1 & 2 & 3 & 4 & 5 \\
\hline$\%$ de bois de tension $\ldots \ldots \ldots$ & $0,71 * * *$ & 0.17 & $0,53 * * *$ & $0,37^{*}$ & $-0,23$ \\
\hline Retrait longitudinal . . & $0,64^{* * * * * *}$ & $0,56 * * *$ & $0,30 *$ & $-0,25$ & 0,31 \\
\hline Densité $\ldots . .$. & 0.28 & $0.73^{* * *}$ & $-0,56 * * *$ & 0,27 & 0,05 \\
\hline Rendement en fibres ... & $0.81 \% \cdots \cdots$ & $-0,03$ & $-0,16$ & $0,47 * * *$ & $-0,27$ \\
\hline Longueur des fibres .. & $0.85 * * *$ & $-0,32 *$ & $-0,23$ & 0,07 & 0,05 \\
\hline Vitesse ultrasons. & $0.75 * * *$ & $-0.52 * * *$ & $-0,12$ & 0,20 & 0,20 \\
\hline
\end{tabular}

La première composante est très liée à toutes les caractéristiques étudićes, sauf la densité du bois; il ne faut sans doute pas accorder trop d'importance au fait que les 2 variables papetières arrivent en tête dans la participation de la $1^{\text {re }}$ composante : elles dépendent en réalité, ainsi qu'on le verra, du pourcentage de bois de tension, et, si elles fournissent des coefficients de corrélation supéricurs, cela peut être dû à ce que la précision des mesures est meilleure pour les rendements et les longueurs de fibres que pour les pourcentages de fibres gélatineuses calculés à l'analyseur d’images.

La deuxième et la troisième composantes se caractérisent surtout par les corrélations, positive pour l'une, négative pour l'autre qui les lient à la densité du bois ; l'analyse en composantes principales apparaît ainsi comme apte à mettre en évidence un effet du facteur étudié sur une propriété du bois dont on sait qu'elle est qualitativement très importante, mais dont le caractère discriminant n'apparaissait pas dans les analyses précédentes.

La projection des 48 échantillons dans lc plan des deux premières composantes est représentée sur la figure 1.

On observe une bonne séparation des 3 catégories de bois, avec, à gauche, les témoins à faible pourcentage de bois de tension, et à faibles valeurs des caractéristiques qui lui sont liées ( $\mathrm{n}^{\circ} 33$ à 48), au centre les échantillons « $P »\left(\mathrm{n}^{*} 17\right.$ à 32), et à droite, les individus à veine verte; quant à la séparation dans le sens de la composante $\mathrm{n}^{\circ} 2$, elle se fait surtout en fonction de la densité du bois. On doit noter également plusieurs groupements de nombres voisins, correspondant à des échantillons d'une même planchette; cela traduit une bonne homogénéité intra-planchette pour l'ensemble des propriétés étudiées.

$\mathrm{Ce}$ sont également des individus tirés dune même planchette initialc (21-22-23, 17-18, 45-46) qui empêchent la ségrégation des 3 groupes d'être parfaite; les 3 premiers correspondent à des échantillons riches en bois de tension et présentant toutes les autres propriétés connexes, mais non colorés en vert ; lexemple inverse étant fourni par les $\mathrm{n}^{\prime s} 1,2$ et 3 , on peut dire que la règle : veine verte $=$ bois de tension est 


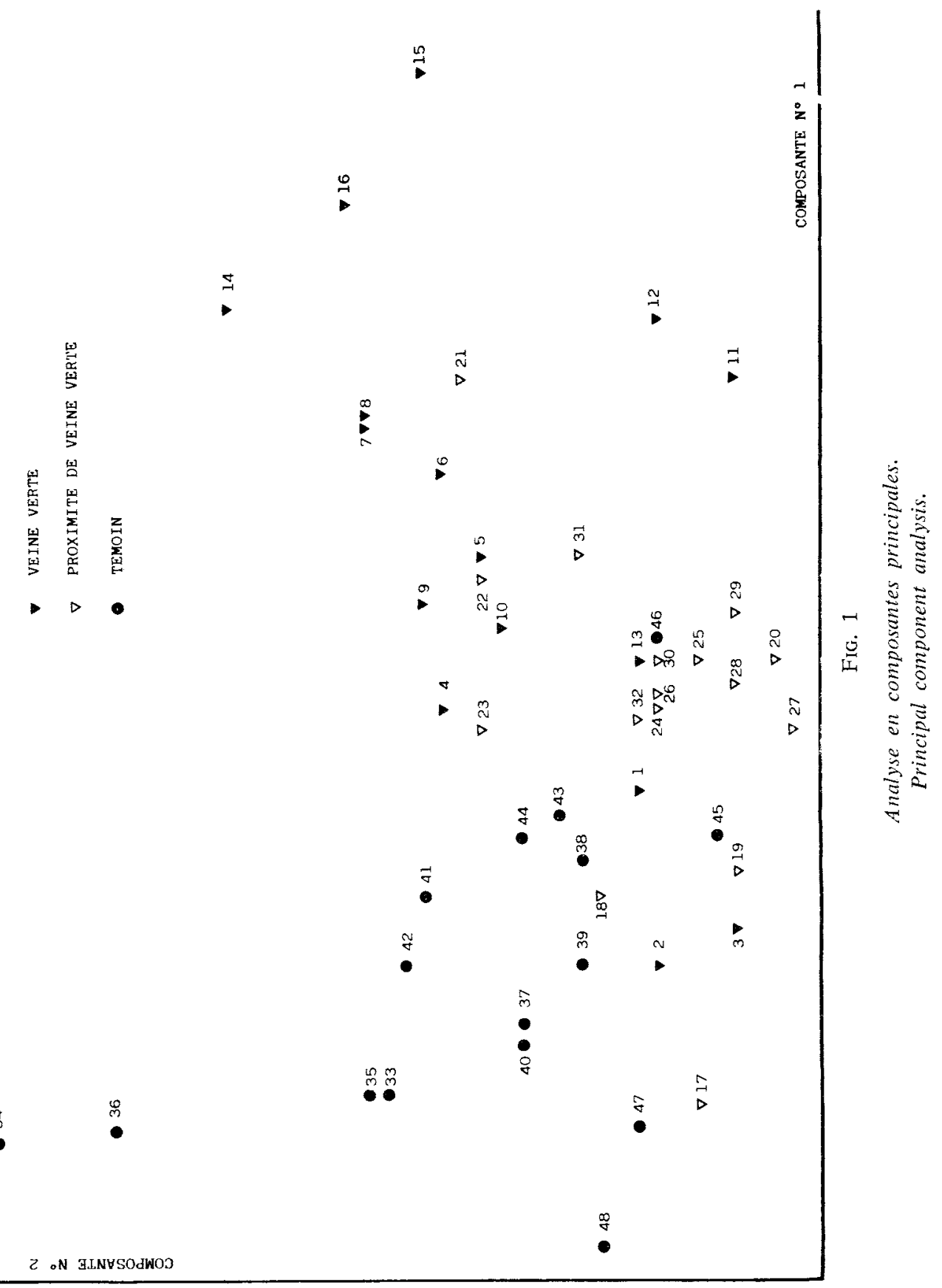


vérifiée de façon générale, mais comporte des exceptions dans les 2 sens. 17 et 18 sont les échantillons hors veine verte jumelés à 1 et 2 ; ils sont décalés dans le même sens par rapport au groupe auquel ils appartiennent. Les $n^{\text {n* }} 45$ et 46 enfin doivent leur situation à la conjonction assez rare d'une densité et d'un pourcentage de bois de tension faibles avec un rendement en fibres, une longueur moyenne de fibres et une vitesse des ultrasons relativement élevés.

\subsection{Corrélations entre variables}

La matrice des coefficients de corréation pour l'ensemble des 48 échantillons est donnée dans le tableau 5.

\section{Tableau 5}

Matrice des coefficients de corrélation.

Table of the correlation coefficients.

\begin{tabular}{|c|c|c|c|c|c|}
\hline & $\begin{array}{l}\text { Bois de } \\
\text { tension }\end{array}$ & Retrait & Densité & Rendement & $\begin{array}{l}\text { Longueur } \\
\text { de fibres }\end{array}$ \\
\hline Retrait longitudinal & $0,55 * * *$ & & & & \\
\hline Densité & 0,13 & $0,35^{*}$ & & & \\
\hline Rendement en fibres & $0,38^{* *}$ & $0,49 * * *$ & 0,18 & & \\
\hline Longueur des fibres & $0,44^{* *}$ & $0,29 *$ & 0,12 & $0,66 * * *$ & \\
\hline Vitesse ultrasons & $0,41^{* *}$ & 0.15 & $-0,04$ & $0,53 * * *$ & $0,77 * * *$ \\
\hline
\end{tabular}

L'exception que constituait la densité du bois au niveau inter-traitement dans les analyses de variances se retrouve ici au niveau de la population totale : c'est la seule caractéristique qui ne soit pas liée aux autres (sauf une corrélation significative au seuil de 5 p. 100 seulement avec le retrait longitudinal); elle apparaît notamment comme statistiquement indépendante du rendement en fibres et de la vitesse des ultrasons, alors que, dans la plupart des expériences, elle croît significativement avec eux.

La liaison la plus étroite, et aussi la plus intéressante parce que jamais signalée jusqu'ici, est celle qui existe entre la vitesse des ultrasons et la longueur des fibres; le coefficient de corrélation atteint même $0,90^{* * *}$ au niveau des moyennes par planchette, et le graphique de la figure 2 montre que celui-ci n'est pas artificiellement gonflé par un point singulier très à l'écart du nuage formé par l'ensemble des autres et présentant des valeurs anormalement fortes ou anormalement faibles pour les deux variables.

On peut penser que les membranes des fibres interviennent comme des guides d'ondes pour les ultrasons dont la vitesse se trouverait réduite à chaque passage d'une fibre à la suivante dans le sens longitudinal, malgré l'efficacité apparente de la liaison assurée par les membranes mitoyennes; à des fibres plus longues correspondraient moins de points de ralentissement par unité de longueur, donc une vitesse plus 


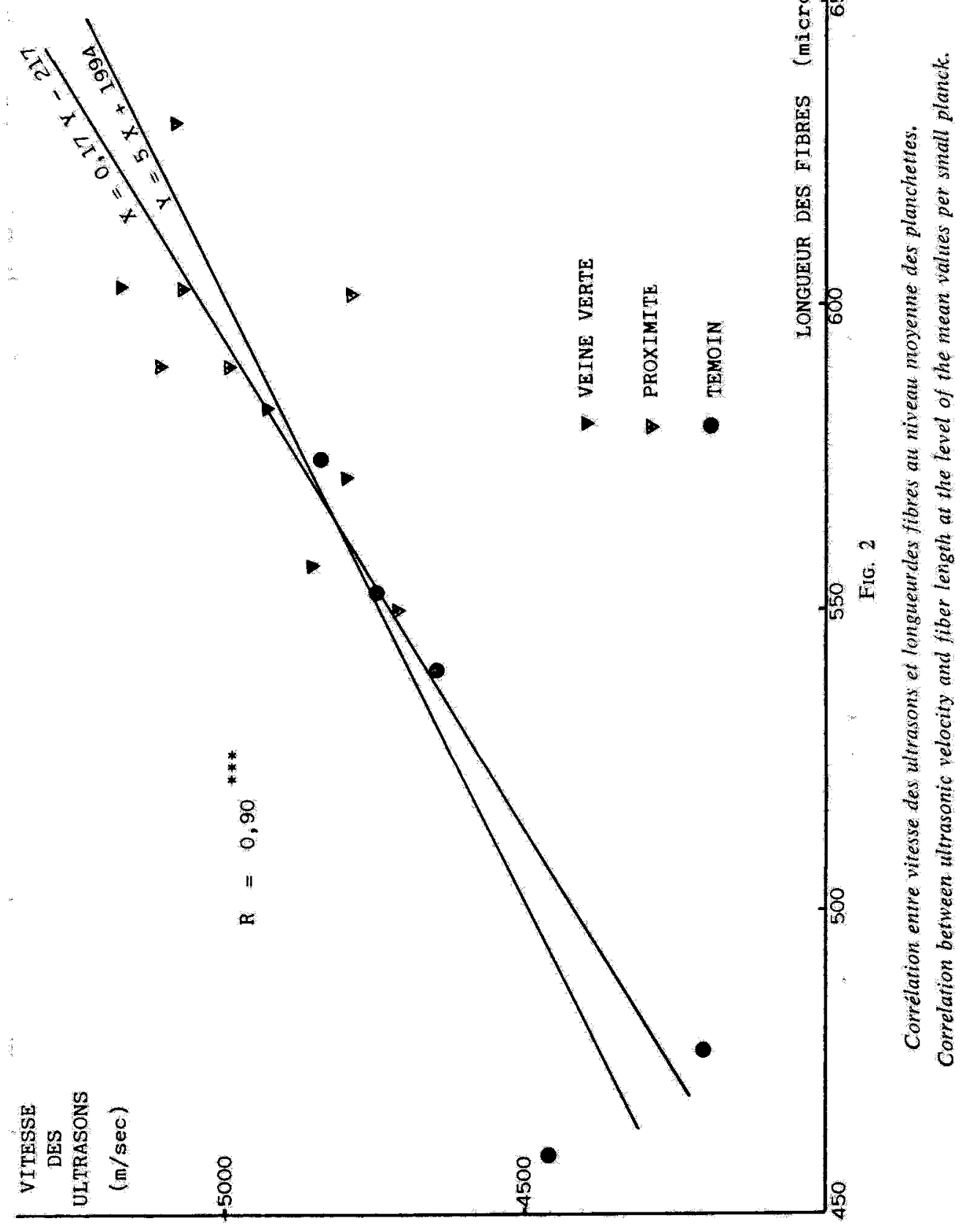


élevée; de la même façon se trouverait expliquée la plus grande vitesse des ultrasons dans le bois à veine verte à fibres plus allongécs que celles du bois normal. On peut noter accessoirement qu'un coefficient aussi élevé confirme la validité des méthodologies originales utilisées pour évaluer les deux paramètres en cause : si l'une ou l'autre donnait des indications plus ou moins aléatoires ou encore entachées d'une forte erreur expérimentale, on ne saurait trouver entre eux qu'indépendance statistique ou, au mieux, que relation faiblement significative (le même raisonnement peut également s'appliquer au pourcentage de bois de tension et au rendement en fibres, caractéristiques dont la mesure, très délicate, a été faite en suivant des méthodes développées à la Station).

Rendement en fibres et longueur de fibres sont liés au seuil de 1 p. 100 au pourcentage de bois de tension, ec qui peut expliquer la part importante quils prennent dans la composante principale $n " 1$.

Bien que les variables apparaissent comme très liées entre elles, des analyses en régression progressive ont été faites pour tenter d’expliquer certaines caractéristiques par l'ensemble des autres :

Pour le retrait longitudinal (RL), lintroduction, après la $1^{\text {r. }}$ variable explicative qui est le pourcentage de bois de tension (BT). successivement du rendement en fibres (R) et de la densité (D) diminue significativement la variance résiduelle. L'équation de la régression est :

$$
\mathrm{RL}=0,196 \mathrm{BT}+0.126 \mathrm{R}+0,047 \mathrm{D}-78,5
$$

et on passe du coefficient de corrélation simple de 0,55 avec la $1^{\text {re }}$ variable à un coefficient de corrélation multiple de 0,67 (on atteint même 0,70 avec la $4^{c}$ variable, la vitesse des ultrasons, dont l'introduction n'est cependant pas significative).

L'estimation du pourcentage de bois de tension à partir du retrait longitudinal (corrélation simple de 0,55 ) est améliorée significativement par lapport de la vitesse des ultrasons (V), en appliquant l'équation :

$$
\mathrm{BT}=1,04 \mathrm{RL}+0,023 \mathrm{~V}-103
$$

avec un coefficient de corrélation multiple de 0.64 .

La vitesse des ultrasons quant à elle ne s"explique que par la seule longucur des fibres, l'apport d'autres variables ne permettant pas d'améliorer significativement son estimation.

\section{Discussion et conclusion}

L'association veine verte - bois de tension apparaît, au vu de l'expérience dont il est rendu compte ici, bien établie, tout au moins au niveau statistique moyen : non seulement la veine verte a bien un pourcentage significalivement supérieur de fibres à couche additionnelle gélatineuse, mais encore elle se différencie par des valeurs plus élevées de 4 caractéristiques lićes au bois de tension : le retrait longitudinal, le rendement et la longueur des fibres, ainsi que la vitesse des ultrasons. 
On peut d'ailleurs noter que certains défauts technologiques reconnus de la veine verte (placages se déformant au séchage, jeux dans les assemblages en cas dutilisation en bois massif) sont assez typiques du bois de tension. MAsset (op. cit.) mentionne même que les merisiers que l'on fait sécher artificiellement par excision de l'aubier sur $2 \mathrm{~cm}$ nauraient jamais de veine verte; or on sait par ailleurs (Guineau \& Chardin, 1973) que la pratique d’annélations circulaires tout à fait semblables est conseillée pour éviter que se dévcloppent, après abaltage, les effets pernicicux des contraintes de croissance, elles-mêmes très liées au bois de tension (Trenard \& Guenliau, 1975 ; FERRAND, 1981); on ne peut pas ne pas faire le rapprochement, même si l'on reste assez sceptique : l'effet mécanique des entailles sur la libération des contraintes ne devrait théoriquement être que très limité ; quant à l'influence qu'elles pourraient avoir sur la veine verte, elle ne devrait logiquement pas s’étendre au bois de tension déjà formé, sauf à supposer que les mécanismes biochimiques susceptibles de transformer les substances colorantes de la veine, ou leurs précurseurs, puissent également stabiliser les membranes des cellules du bois plus ancien; des recherches sur l'identification, la biosynthèse et la biodégradation des composés chimiques de la veine verte seraient à cet égard bien utiles.

Il faut cependant rappeler ici que veine verte et bois de tension ne sont pas toujours synonymes au niveau individuel, puisqu'on trouve aussi bien des échantillons très caractéristiques quant à leur couleur, mais à très faible pourcentage de fibres de tension et à retrait longitudinal modéré, que des témoins à valeurs élevées pour ces 2 critères.

Le problème du bois «intermédiaire » reste par ailleurs posé : on a vu que, bien que prélevé hors zone verte mais à son voisinage, il ne s'en différencic pas pour le rendement en fibres, la longueur des fibres et la vitesse des ultrasons, alors qu'il est au contraire très proche des témoins quant au retrait longitudinal : le fait que les échantillons « $\mathbf{P}$ » ą́ent un pourcentage de bois de tension intermédiaire entre ceux de la veine verte et des témoins laisse à penser à l'existence d'un seuil en deçà duquel la rétractibilité resterait modérée et la coloration verte ne se produirait pas. Par ailleurs, l'existence de bois possédant certaines propriétés intermédiaires entre celles du bois normal ct celles du bois de tension a été signalée au voisinage de ce dernier : sur le hêtre, Gonet (1971) a montré qu’il se caractérisait par des vaisseaux moins nombreux et de plus petit diamètre et par une plus grande densité de rayons ligneux ; ces éléments n'ont pas pu être mesurés sur les merisiers au grossissement utilisé à l'analyseur d'images; si un phénomène analogue se produisait dans les arbres de cette espèce atteints de veine verte, il pourrait expliquer que certaines propriétés du bois soient affectées et d'autres non.

Cependant, si l'on raisonne au niveau de populations de merisiers, on peut dire que tout traitement (au sens large) ayant pour cffet de réduire le bois de tension diminuera corrélativement le risque de veine verte : bien que cette espèce forme rarement des peuplements homogènes sur des surfaces importantes, le forestier a parfois à choisir, lors des opérations sylvicoles, entre plusieurs individus poussant côte à côte ; il doit alors éliminer préférentiellement les arbres à tige penchée ou flexueuse, générateurs de bois de tension, quitte à en conserver d'autres à fût plus court ou fourchus à mi-hauteur, mais rectilignes et verticaux.

Enfin, toute possibilité de déterminisme génétique du bois de tension n'est pas à exclure (on connaît déjà la très grande variabilité entre clones de la production de 
bois «pelucheux», c’est-à-dire de bois de tension, chez le peuplier). Dans lhypothèse où il en serait de même pour le merisier, il serait raisonnable, au moment où l'on envisage la création de variétés multiclonales de cette essence par bouturage de drageons, voire même par cultures in vitro (CoRNu, communication personnelle) de chercher à mettre au point des tests précoces d'estimation du bois de tension, à en étudier les corrélations jeune-adulte et à en calculer l'héritabilité au sens large.

Il conviendra aussi, alors quiun important effort de plantation de merisiers est entrepris pour satisfaire les besoins de l'industrie nationale, d'étudier linfluence de la station sur la fréquence de veines vertes, si l'on veut réellement atteindre le but recherché, c'est-à-dire assurer un approvisionnement satisfaisant en bois de qualité placage ou ébénisterie.

\section{Remerciements}

De nombreux membres de la Station de Recherches sur la Qualité du Bois ont collaboré à cette étude, qui a intéressé plusieurs laboratoires, et notamment :

- Simone Garros, pour les mesures de retrait, les microcuissons, et la réalisation des coupes microscopiques,

- Françoise Huber, pour la mise au point d'un programme spécifique pour l'analyseur d'images, et pour les analyses elles-mêmes (conjointement avec P. GelHaYe),

- Voïchita Bucur, pour les mesures de vitesses des ondes ultrasonores,

- Yolande NiKolski, pour les déterminations des longueurs de fibres,

- Danièle Aubert, pour les analyses statistiques.

Que tous en soient ici vivement remerciés!

\section{Summary}

Tentative characterization of green vein in cherry tree.

Sixteen samples of green vein (V) 16 samples close to the green vein (P) and 16 controls are studied. The samples $V$ have a higher percentage of tension wood, and also differ by higher values of longitudinal shrinkage, pulp yield, fiber length and velocity of ultrasonic waves. The samples $\mathbf{P}$ have intermediate values, but are more similar to the samples $V$ for pulp yield, fiber length and ultrasonic velocity, and on the contrary to the controls for shrinkage. Wood density is not a discriminant criterion, and is independant of the other characteristics that are, on the other hand, strongly correlated to each other, particularly the fiber length and the ultrasonic velocity. Green vein and tension wood are associated at the statistical level, but there are exceptions in both directions at the individual level.

$$
\begin{array}{r}
\text { Reçu le } 8 \text { août } 1982 . \\
\text { Accepté le } 13 \text { janvier } 1983 .
\end{array}
$$

\section{Références bibliographiques}

Bucur V., 1981. Détermination du module d'Young du bois par une méthode dynamique sur carottes de sondage. Ann. Sci. For., 38 (2), 283-298.

FERRAND J.Ch., 1981. Recherches des solutions pratiques à apporter aux problèmes posés par les contraintes de croissance des arbres forestiers. Thèse de Docteur-Ingénieur en Sciences du Bois, INPL, 11 décembre 1981. 
FERrand J.Ch., 1983. La veine verte du Merisier: est-ce du bois de tension? Rev. For. Fr., n" 2, 85-87.

Gonet B., 1971. Some technical properties of the tension wood of beach. Sy/wan, 6, 25-35.

Gueneau P., Chardin A., 1973. Les contraintes de croissance. Cah. Centre Tech. For. Trop.. 3, 1973.

Hughes F.E., 1965. Tension Wood. A review of literature. For. Abstr., 26 (1), 2-9.

JANIN G., 1972. Microcuissons papetières. Méthode adaptée aux recherches forestières portant sur la détermination des caractéristiques papetières individuelles sur arbres vivants à l'aide d'échantillons de bois dont le mode de prélèvement, l'aspect et le poids ne sont pas usuels. La Papeterie, 3, 1972.

JANin G., 1981. Mesure automatique en continu de la longueur des fibres. Rapport de firi de contrat C.E.E. n" 021-79-RCPF.

Masset P.L., 1977. Etude des liaisons entre la qualité technologique du bois de merisier et la station. Rapport E.N.G.R.E.F., avril-mai 1977, 1-19.

Trenard Y., Gueneau P., 1975. Relations entre contraintes de croissance longitudinales et bois de tension dans le hêtre. Holzforschung, 29 (6), 217-223. 